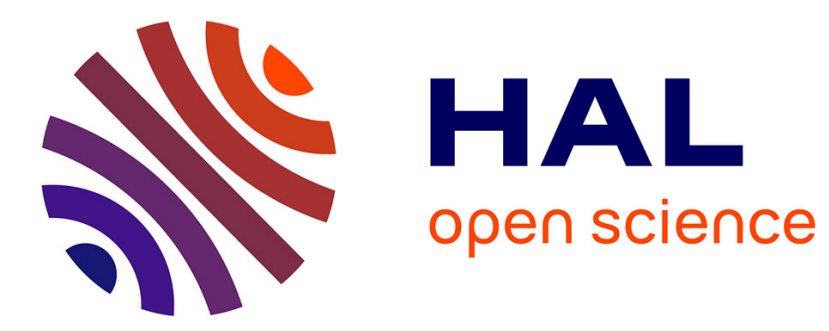

\title{
Robust adaptive stabilization by delay under state parametric uncertainty and measurement bias
}

Jian Wang, Stanislav Aranovskiy, Emilia Fridman, Dmitry Sokolov, Denis Efimov, Alexey Bobtsov

\section{- To cite this version:}

Jian Wang, Stanislav Aranovskiy, Emilia Fridman, Dmitry Sokolov, Denis Efimov, et al.. Robust adaptive stabilization by delay under state parametric uncertainty and measurement bias. IEEE Transactions on Automatic Control, 2021, 66 (11), pp.5459-5466. 10.1109/TAC.2020.3045125 . hal03059893

\section{HAL Id: hal-03059893 https://hal.inria.fr/hal-03059893}

Submitted on 13 Dec 2020

HAL is a multi-disciplinary open access archive for the deposit and dissemination of scientific research documents, whether they are published or not. The documents may come from teaching and research institutions in France or abroad, or from public or private research centers.
L'archive ouverte pluridisciplinaire HAL, est destinée au dépôt et à la diffusion de documents scientifiques de niveau recherche, publiés ou non, émanant des établissements d'enseignement et de recherche français ou étrangers, des laboratoires publics ou privés. 


\title{
Robust adaptive stabilization by delay under state parametric uncertainty and measurement bias
}

\author{
Wang J., Aranovskiy S., Fridman E., Sokolov D., Efimov D., Bobtsov A.A.
}

\begin{abstract}
An output robust adaptive control is designed for a class of Lipschitz nonlinear systems under assumption that the measurements are available with a constant bias and the state equations linearly parameterized by unknown parameters and external disturbances. A dynamic state reconstruction (synthesis of an observer) is avoided by using delayed values of the output in the feedback and adaptation laws. The analysis of robust stability for the resulted time-delay system is performed by using the Lyapunov-Krasovskii approach. The control and adaptation gains can be selected as a solution of the proposed linear matrix inequalities. This research is motivated by a nonlinear pendulum control problem, and the efficacy of the developed control is demonstrated on this application through experiments.
\end{abstract}

\section{INTRODUCTION}

Design of identification algorithms, estimators and regulators for dynamical systems are fundamental and complex problems studied in the control theory. Since in all engineering applications the models of the plants are subjected by uncertainty of different kinds (e.g., exogenous unknown inputs, measurement noises and perturbations, unknown parameters or unmodeled dynamics), the design and analysis methods are classified by their abilities to undertake the plant's incertitude [1]. In particular, the group of methods belonging to the adaptive control theory deals with compensation of influence on estimation and stabilization of unknown parameters [2].

In many cases, due to information transmission in the input-output channels, delays appear in the dynamics of the controlled plant [3], [4]. Influence of a delay on the system stability is vital in many cases [5], [6], and it usually leads to degradation of the performances of regulation or estimation [6]. However, in some cases introduction of a delay may result in an improvement of the system transients (see [7], [8], [9], [10], [11] and the references therein). The idea of these papers is that unmeasured components of the state can be calculated using delayed values of the measured variables, which allow a design of observer to be passed by, but at the price of a more sophisticated stability analysis (that is based in these works on

This work was partially supported by 111 project No. D17019 (China), by the Government of Russian Federation (Grant 08-08), by the Ministry of Science and Higher Education of Russian Federation, passport of goszadanie no. 2019-0898 and by Israel Science Foundation (grant No 673/19).

Wang J. is with Hangzhou Dianzi University, Hangzhou, China.

Aranovskiy S. is with CentaleSupélec -IETR, Avenue de la Boulaie, 35576 Cesson-Sévigné, France.

Fridman E. is with School of Electrical Engineering, Tel-Aviv University, Tel-Aviv 69978, Israel.

Sokolov D. is with Université de Lorraine, Inria, CNRS, LORIA, F-54000 Nancy, France.

Efimov D. is with Inria, Univ. Lille, CNRS, UMR 9189 - CRIStAL, F59000 Lille, France.

Bobtsov A.A. is with ITMO University, 197101 Saint Petersburg, Russia. an appropriate Lyapunov-Krasovskii functional, and another possible approach is the Lyapunov-Razumikhin method; and the complexity comes from the fact that the closed-loop system becomes unstable in the delay-free case).

The goal of this note is to extend the results obtained in [8], [9] for linear systems to adaptive stabilization of a class of nonlinear systems, which include a globally Lipschitz nonlinearity, unknown parameters, exogenous disturbances, and have a part of the measurements available with a constant bias, which is induced by a sensor error (a preliminary version of this study appears in [12] without proofs and additional details). Since for embedded control and estimation solutions, the amount of computations needed for realization is a critical resource (more important than the used memory in some scenarios), in this note we avoid to design an (reduced order) observer for the state, but introduce delayed measurements in the feedback and adaptation algorithm. The closed-loop system becomes time-delayed, then stability analysis of the regulation error is based on the Lyapunov-Krasovskii functional proposed in [8], [9], whose properties can be assessed by investigating linear matrix inequalities (LMIs).

It is important to note that there exist papers devoted to adaptive control of time-delay systems as, for example, [13], [14], [15] (the uncertain parameters appear in the state equation only), or papers dealt with adaptive/robust control for systems with multiplicative uncertain parameters in the output equation [16], [17] (without presence of time delays) or [18], but to the best of our knowledge there is no theory dealing with time-delay systems subjected to parametric uncertainty in the state dynamics and the output measurements simultaneously.

The selected problem statement is not artificial, since it can be motivated by a mechanical balancing system application, e.g., stabilization of a walking robot, where a constant bias distorts the angular position measurements. This application is described with more details in [19], where the authors proposed a nonlinear bias and velocity estimator proving local asymptotic convergence. In this paper, we are solving the same problem by designing a delay-based adaptive algorithm, which stabilizes the pendulum in the presence of the measurement bias 1 . We also experimentally verify the proposed solution with the inverted pendulum hardware setup equivalent to the one used in [19].

The outline of this article is as follows. The preliminaries are given in Section III The problem statement is introduced in Section III. The adaptive control design and stability con-

\footnotetext{
${ }^{1}$ The preliminary version of this work [12] does not contain proofs and experiments, the main assumption is relaxed in this paper.
} 
ditions are presented in Section IV A nonlinear pendulum application is considered in Section $\mathrm{V}$

\section{PRELIMINARIES}

Denote by $\mathbb{R}$ the set of real numbers and $\mathbb{R}_{+}=\{s \in$ $\mathbb{R}: s \geq 0\}$. For a Lebesgue measurable function of time $d:[a, b] \rightarrow \mathbb{R}^{m}$, where $-\infty \leq a<b \leq+\infty$, define the norm $\|d\|_{[a, b)}=\operatorname{ess} \sup _{t \in[a, b)}|d(t)|$, where $|\cdot|$ is the standard Euclidean norm in $\mathbb{R}^{m}$, then $\|d\|_{\infty}=\|d\|_{[0,+\infty)}$ and the space of $d$ with $\|d\|_{[a, b)}<+\infty\left(\|d\|_{\infty}<+\infty\right)$ we further denote as $\mathcal{L}_{[a, b]}^{m}\left(\mathcal{L}_{\infty}^{m}\right)$.

Denote by $C_{[a, b]}^{n}, a, b \in \mathbb{R}$ the Banach space of continuous functions $\phi:[a, b] \rightarrow \mathbb{R}^{n}$ with the uniform norm $\|\phi\|_{[a, b]}=\sup _{a \leq s \leq b}|\phi(s)|$; and by $\mathbb{W}_{[a, b]}^{1, \infty}$ the Sobolev space of absolutely continuous functions $\phi:[a, b] \rightarrow \mathbb{R}^{n}$ with the norm $\|\phi\|_{\mathbb{W}}=\|\phi\|_{[a, b)}+\|\dot{\phi}\|_{[a, b)}<+\infty$, where $\dot{\phi}(s)=\frac{\partial \phi(s)}{\partial s}$ is a Lebesgue measurable essentially bounded function, i.e. $\dot{\phi} \in \mathcal{L}_{[a, b]}^{n}$.

A continuous function $\sigma: \mathbb{R}_{+} \rightarrow \mathbb{R}_{+}$belongs to class $\mathcal{K}$ if it is strictly increasing and $\sigma(0)=0$; it belongs to class $\mathcal{K}_{\infty}$ if it is also radially unbounded. A continuous function $\beta: \mathbb{R}_{+} \times \mathbb{R}_{+} \rightarrow \mathbb{R}_{+}$belongs to class $\mathcal{K} \mathcal{L}$ if $\beta(\cdot, r) \in \mathcal{K}$ and $\beta(r, \cdot)$ is decreasing to zero for any fixed $r>0$.

The symbol $\overline{1, m}$ is used to denote a sequence of integers $1, \ldots, m$. For a symmetric matrix $P \in \mathbb{R}^{n \times n}$, the minimum and the maximum eigenvalues are denoted as $\lambda_{\min }(P)$ and $\lambda_{\max }(P)$, respectively. For a matrix $A \in \mathbb{R}^{n \times m},|A|=$ $\sqrt{\lambda_{\max }\left(A^{\top} A\right)}$ is the induced norm. The identity matrix of dimension $n \times n$ is denoted by $I_{n}$.

\section{A. Neutral time-delay systems}

Consider an autonomous functional differential equation of neutral type with inputs [20]:

$$
\dot{x}(t)=f\left(x_{t}, \dot{x}_{t}, d(t)\right)
$$

for almost all $t \geq 0$, where $x(t) \in \mathbb{R}^{n}$ and $x_{t} \in \mathbb{W}_{[-\tau, 0]}^{1, \infty}$ is the state function, $x_{t}(s)=x(t+s),-\tau \leq s \leq 0$, with $\dot{x}_{t} \in \mathcal{L}_{[-\tau, 0]}^{n} ; d(t) \in \mathbb{R}^{m}$ is the external input, $d \in \mathcal{L}_{\infty}^{m}$; $f: \mathbb{W}_{[-\tau, 0]}^{1, \infty} \times \mathcal{L}_{[-\tau, 0]}^{n} \times \mathbb{R}^{m} \rightarrow \mathbb{R}^{n}$ is a continuous functional, that is globally Lipschitz in the second variable with a constant smaller than 1, ensuring forward uniqueness and existence of the system solutions [20]. We assume $f(0,0,0)=0$. For the initial function $x_{0} \in \mathbb{W}_{[-\tau, 0]}^{1, \infty}$ and disturbance $d \in \mathcal{L}_{\infty}^{m}$ denote a unique solution of the system (17) by $x\left(t, x_{0}, d\right)$, which is an absolutely continuous function of time defined on some maximal interval $[-\tau, T)$ for $T>0$, then $x_{t}\left(x_{0}, d\right) \in \mathbb{W}_{[-\tau, 0]}^{1, \infty}$ represents the corresponding state function with $x_{t}\left(s, x_{0}, d\right) \stackrel{=}{=}$ $x\left(t+s, x_{0}, d\right)$ for $-\tau \leq s \leq 0$.

Given a locally Lipschitz continuous functional $V: \mathbb{R} \times$ $\mathbb{W}_{[-\tau, 0]}^{1, \infty} \times \mathcal{L}_{[-\tau, 0]}^{n} \rightarrow \mathbb{R}_{+}$define its derivative in the Driver's form:

$$
\begin{aligned}
D^{+} V(t, \phi, d)=\lim _{h \rightarrow 0^{+}} & \sup \frac{1}{h}\left[V\left(t+h, x_{h}(\phi, \tilde{d}), \dot{x}_{h}(\phi, \tilde{d})\right)\right. \\
& -V(t, \phi, \dot{\phi})],
\end{aligned}
$$

where $x_{h}(\phi, \tilde{d})$ is a solution of the system $(1)$ for $\phi \in \mathbb{W}_{[-\tau, 0]}^{1, \infty}$ and $\tilde{d}(t)=d$ for all $t \geq 0$ and some $d \in \mathbb{R}^{m}$.

\section{B. ISS of time delay systems}

The input-to-state stability (ISS) property is an extension of the conventional stability paradigm to the systems with external inputs [21], [22], [23].

Definition 1. [22], [23] The system (1) is called practical ISS, if for all $x_{0} \in \mathbb{W}_{[-\tau, 0]}^{1, \infty}$ and $d \in \mathcal{L}_{\infty}^{m}$ there exist $q \geq 0, \beta \in \mathcal{K} \mathcal{L}$ and $\gamma \in \mathcal{K}$ such that

$$
\left|x\left(t, x_{0}, d\right)\right| \leq \beta\left(\left\|x_{0}\right\|_{\mathbb{W}}, t\right)+\gamma\left(\|d\|_{\infty}\right)+q \quad \forall t \geq 0 .
$$

If $q=0$ then (1) is called ISS.

For establishment of this stability property, the LyapunovKrasovskii theory can be used [22], [23], [10].

Definition 2. A locally Lipschitz continuous functional $V$ : $\mathbb{R}_{+} \times \mathbb{W}_{[-\tau, 0]}^{1, \infty} \times \mathcal{L}_{[-\tau, 0]}^{n} \rightarrow \mathbb{R}_{+}$(i.e., $V(t, \phi, \dot{\phi})$ ) is called simple if $D^{+} V(t, \phi, d)$ is independent on $\ddot{\phi}$.

For instance, a locally Lipschitz functional $V: \mathbb{R}_{+} \times$ $\mathbb{W}_{[-\tau, 0]}^{1, \infty} \rightarrow \mathbb{R}_{+}$is simple, another example of a simple functional is given in Theorem 8 below.

Definition 3. A locally Lipschitz continuous functional $V$ : $\mathbb{R}_{+} \times \mathbb{W}_{[-\tau, 0]}^{1, \infty} \times \mathcal{L}_{[-\tau, 0]}^{n} \rightarrow \mathbb{R}_{+}$is called practical ISS Lyapunov-Krasovskii functional for the system (1) if it is simple and there exist $r \geq 0, \alpha_{1}, \alpha_{2} \in \mathcal{K}_{\infty}$ and $\alpha, \chi \in \mathcal{K}$ such that for all $t \in \mathbb{R}_{+}, \phi \in \mathbb{W}_{[-\tau, 0]}^{1, \infty}$ and $d \in \mathbb{R}^{m}$ :

$$
\begin{gathered}
\alpha_{1}(|\phi(0)|) \leq V(t, \phi, \dot{\phi}) \leq \alpha_{2}\left(\|\phi\|_{\mathbb{W}}\right), \\
V(t, \phi, \dot{\phi}) \geq \max \{r, \chi(|d|)\} \Longrightarrow D^{+} V(t, \phi, d) \leq-\alpha(V(t, \phi, \dot{\phi})) .
\end{gathered}
$$

If $r=0$ then $V$ is an ISS Lyapunov-Krasovskii functional.

Theorem 4. [23] If there exists a (practical) ISS LyapunovKrasovskii functional for the system (1), then it is (practical) ISS with $\gamma=\alpha_{1}^{-1} \circ \chi$.

Converse results for Theorem 4 can be found in [24], [25].

\section{RoBust OUTPUT ADAPTIVE REGULATION WITH BIASED MEASUREMENTS}

Consider a nonlinear system for the time $t \geq 0$ :

$$
\begin{aligned}
\dot{x}_{1}(t)= & x_{2}(t), \\
\dot{x}_{2}(t)= & A_{21} x_{1}(t)+A_{22} x_{2}(t)+A_{23} x_{3}(t) \\
& +B_{1}\left(u(t)+\Omega(t) \theta_{2}\right)+L_{1} \phi(x(t))+d_{1}(t), \\
\dot{x}_{3}(t)= & A_{31} x_{1}(t)+A_{32} x_{2}(t)+A_{33} x_{3}(t) \\
& +B_{2}\left(u(t)+\Omega(t) \theta_{2}\right)+L_{2} \phi(x(t))+d_{2}(t), \\
y_{1}(t)= & x_{1}(t)+\theta_{1}, \quad y_{2}(t)=x_{3}(t),
\end{aligned}
$$

where $x_{1}(t) \in \mathbb{R}^{n}$ and $x_{2}(t) \in \mathbb{R}^{n}$ are the position and velocity, respectively, $x_{3}(t) \in \mathbb{R}^{p}$ is an additional state, $x(t)=\left[\begin{array}{lll}x_{1}^{\top}(t) & x_{2}^{\top}(t) & x_{3}^{\top}(t)\end{array}\right]^{\top} \in \mathbb{R}^{2 n+p}$ is the total state vector of (2), the initial conditions $x(0)=x_{0} \in \mathbb{R}^{2 n+p}$ are unknown; $u(t) \in \mathbb{R}^{m}$ is the control input; $y(t)=$ $\left[y_{1}^{\top}(t) y_{2}^{\top}(t)\right]^{\top} \in \mathbb{R}^{n+p}$ is the output available for measurements, $d(t)=\left[d_{1}^{\top}(t) d_{2}^{\top}(t)\right]^{\top} \in \mathbb{R}^{n+p}$ is the disturbance with $d \in \mathcal{L}_{\infty}^{n+p} ; \theta_{1} \in \mathbb{R}^{n}$ is the vector of biases in the measurements of the position $x_{1}(t), \theta_{2} \in \mathbb{R}^{r}$ is the vector of uncertain parameters in the state dynamics, $\theta=\left[\begin{array}{ll}\theta_{1}^{\top} & \theta_{2}^{\top}\end{array}\right]^{\top} \in \mathbb{R}^{n+r}$, 
the regressor $\Omega: \mathbb{R}_{+} \rightarrow \mathbb{R}^{m \times r}$ is a known continuous matrix function; the nonlinearity $\phi$ can be partitioned as

$$
\phi(x)=\left[\begin{array}{c}
\phi_{1}(x) \\
\phi_{2}\left(x_{3}\right)
\end{array}\right],
$$

where $\phi_{1}: \mathbb{R}^{2 n+p} \rightarrow \mathbb{R}^{s_{1}}$ and $\phi_{2}: \mathbb{R}^{p} \rightarrow \mathbb{R}^{s_{2}}$, then we can decompose $L_{1}=\left[\begin{array}{ll}L_{11} & L_{12}\end{array}\right], L_{2}=\left[\begin{array}{ll}L_{21} & L_{22}\end{array}\right]$, and $\phi(x)$ is assumed to be Lipschitz continuous; all the matrices are constant and known having the corresponding dimensions.

Therefore, the considered system is subjected by unknown inputs $d(t)$, it contains unknown parameters $\theta$ (part of them corrupt the measurements), and the equations of (2) are nonlinear and time-varying due to the presence of $\phi$ and $\Omega$, respectively. Many mechanical systems (subsystem with coordinates $x_{1}$ and $x_{2}$ ) in connection with a motor, sensor or actuator (the variable $x_{3}$ ) can be represented in the form (2).

The goal is to design an (dynamical) output control input $u(t)=u\left(y_{1}(t), y_{2}(t), \Omega(t)\right)$ ensuring a practical ISS property of the closed loop system for all $x_{0} \in \mathbb{R}^{2 n+p}$ and all $d \in \mathcal{L}_{\infty}^{n+p}$ under the restriction to minimize the computational complexity of the algorithm (in order to be able to use the proposed solution as a component of an embedded system).

Remark 5. Note that a change of variables $\tilde{x}_{1}(t)=x_{1}(t)+\theta_{1}$, $\tilde{x}_{i}(t)=x_{i}(t)$ for $i=2,3$ transforms (2) to a dynamics with two purely measured states,

$$
y_{1}(t)=\tilde{x}_{1}(t), y_{2}(t)=\tilde{x}_{3}(t),
$$

but it makes the equations nonlinearly parameterized by $\theta_{1}$. Extending this state by $\tilde{x}_{4}(t)=\theta_{1}$ poses in question the observability of such an augmented system with $\dot{\tilde{x}}_{4}(t)=0$, which becomes dependent on the characteristics of $\phi$.

We need the following hypothesis on the properties of (2):

Assumption 1. For the regressor function $\Omega(t)$ there is a known upper bound $\bar{\Omega} \geq 0$ such that $\sup _{t>0}|\Omega(t)| \leq \bar{\Omega}$.

The function $\phi$ satisfies a sector condition $\phi^{\top}(x) \phi(x) \leq$ $x^{\top} \Upsilon x$ for all $x \in \mathbb{R}^{2 n+p}$ and a symmetric matrix $0 \leq \Upsilon \in$ $\mathbb{R}^{(2 n+p) \times(2 n+p)}$. The function $\phi_{2}: \mathbb{R}^{p} \rightarrow \mathbb{R}^{s_{2}}$ is known.

Under the introduced restriction on $\phi$ the system (2) has well-defined solutions for all $t \geq 0$ for any $x_{0} \in \mathbb{R}^{2 n+p}$ and any $d \in \mathcal{L}_{\infty}^{n+p}[1]$.

Remark 6. The regressor $\Omega$ can be a nonlinear function of any measured information, e.g., $\Omega(t)=\Omega\left(t, y_{1}(t), y_{2}(t), u(t)\right)$, provided that it is globally bounded.

\section{MAin RESUlts}

Due to a rather complicated structure of the considered system and introduced uncertainty, clearly, for realization of a robustly stabilizing control it is necessary to use the full state $x(t)$ information. Consequently, it is required to design an estimator for $x_{1}(t)$, which is measured with an unknown bias $\theta_{1}$, and for its velocity $x_{2}(t)$. Facing all uncertain terms presented in (2), i.e. $\theta_{2}$ and $d(t)$, such an observation problem becomes rather intriguing, and a corresponding observer solving these issues will be also complex and nonlinear. In [7], [8], [9] an approach is presented for design of a linear delayed output static control for a linear system, which avoids a state observer design by introducing the estimates of $x(t)$ through delayed output $y(t)$ values. Hence, such a method has a low computational capacity (since for delay operation only memory is needed). In this work we will follow the same approach.

Defining $x_{1}(t-h)=x_{1}(0)$ for $t \in[0, h]$, where $h>0$ is the delay, the control algorithm proposed in this paper is

$$
\begin{aligned}
u(t)= & -\left(K_{1}+K_{2}\right) y_{1}(t)+K_{2} y_{1}(t-h) \\
& -K_{3} y_{2}(t)-K_{4} \phi_{2}\left(y_{2}(t)\right)+K_{1} \hat{\theta}_{1}(t)-\Omega(t) \hat{\theta}_{2}(t),
\end{aligned}
$$

where $\hat{\theta}_{1}(t) \in \mathbb{R}^{n}$ and $\hat{\theta}_{2}(t) \in \mathbb{R}^{r}$ are the estimates of $\theta_{1}$ and $\theta_{2}$, respectively; $K_{i} \in \mathbb{R}^{m \times n}$ for $i=1,2, K_{3} \in \mathbb{R}^{m \times p}$ and $K_{4} \in \mathbb{R}^{m \times s_{2}}$ are the control gains to be derived. Similarly, an adaptive law for $\hat{\theta}_{1}(t)$ can be synthesized:

$$
\begin{aligned}
\dot{\hat{\theta}}_{1}(t)= & \left(F_{1}+F_{2}\right) y_{1}(t)-F_{2} y_{1}(t-h) \\
& +F_{3} y_{2}(t)+F_{4} \phi_{2}\left(y_{2}(t)\right)-F_{1} \hat{\theta}_{1}(t),
\end{aligned}
$$

where $F_{i} \in \mathbb{R}^{n \times n}$ for $i=1,2, F_{3} \in \mathbb{R}^{n \times p}$ and $F_{4} \in \mathbb{R}^{n \times s_{2}}$ are the adaptation gains which will be defined later. An adaptive law for $\hat{\theta}_{2}(t)$ is more sophisticated, and such a choice of the structure will become clear from the stability analysis given next:

$$
\begin{aligned}
\dot{\hat{\theta}}_{2}(t)= & \Omega^{\top}(t)\left[\left(S_{1}+S_{2}\right) y_{1}(t)-S_{2} y_{1}(t-h)\right. \\
& \left.+S_{3} y_{2}(t)+S_{4} \phi_{2}\left(y_{2}(t)\right)-S_{1} \hat{\theta}_{1}(t)\right]-S_{5} \hat{\theta}_{2}(t),
\end{aligned}
$$

where $S_{i} \in \mathbb{R}^{m \times n}$ for $i=1,2, S_{3} \in \mathbb{R}^{m \times p}, S_{4} \in \mathbb{R}^{m \times s_{2}}$ and $S_{5} \in \mathbb{R}^{r \times r}$ are also the adaptation gains.

Remark 7. There is also an algebraic way to solve the problem of estimation of unknown values $\theta_{1}, \theta_{2}$ and signals $x_{1}(t)$, $x_{2}(t)$ (in the framework of indirect adaptive control), which is based on some structural restrictions and auxiliary filtering. Indeed, let $p=n$ and $J_{1} L_{11}=J_{2} L_{21}$ for some matrices $J_{1}$ and $J_{2}$, then define $\zeta(t)=J_{1} x_{2}(t)-J_{2} x_{3}(t)$ with

$$
\begin{gathered}
\dot{\zeta}(t)=Y_{1}\left(y_{1}(t)-\theta_{1}\right)+Y_{2} \dot{y}_{1}(t)+Y_{3} y_{2}(t)+Y_{4}(u(t) \\
\left.+\Omega(t) \theta_{2}\right)+Y_{5} \phi_{2}\left(y_{2}(t)\right)+J_{1} d_{1}(t)-J_{2} d_{2}(t)
\end{gathered}
$$

where

$$
\begin{gathered}
Y_{1}=J_{1} A_{21}-J_{2} A_{31}, Y_{2}=J_{1} A_{22}-J_{2} A_{32}, \\
Y_{3}=J_{1} A_{23}-J_{2} A_{33}, \\
Y_{4}=J_{1} B_{1}-J_{2} B_{2}, Y_{5}=J_{1} L_{12}-J_{2} L_{22} .
\end{gathered}
$$

Let also for brevity $d_{1}(t)=0, d_{2}(t)=0, Y_{1} \neq 0$. Note that by construction $\dot{\zeta}(t)=J_{1} \ddot{y}_{1}(t)-J_{2} \dot{y}_{2}(t)$, and equating the expressions for $\zeta(t)$ we obtain:

$$
\tilde{\zeta}(t)=-Y_{1} \theta_{1}+Y_{4} \tilde{\Omega}(t) \theta_{2},
$$

which is a linear regressor model with respect to unknown parameters $\theta_{1}$ and $\theta_{2}$ that can be used for their identification, where $\tilde{\zeta}(t)$ and $\tilde{\Omega}(t)$ variables calculated as

$$
\begin{gathered}
\tilde{\Omega}(t)=\frac{\lambda^{2}}{(s+\lambda)^{2}} \Omega(t), \tilde{\zeta}(t)=J_{1} \frac{\lambda^{2} s^{2}}{(s+\lambda)^{2}} y_{1}(t) \\
-Y_{2} \frac{\lambda^{2} s}{(s+\lambda)^{2}} y_{1}(t)-J_{2} \frac{\lambda^{2} s}{(s+\lambda)^{2}} y_{2}(t) \\
-\frac{\lambda^{2}}{(s+\lambda)^{2}}\left[Y_{1} y_{1}(t)+Y_{3} y_{2}(t)+Y_{4} u(t)+Y_{5} \phi_{2}\left(y_{2}(t)\right)\right]
\end{gathered}
$$


with $s$ being the differentiating operator and $\lambda>0$ is a tuning parameter of the filters. Inversely, if $d_{1}(t)=0, d_{2}(t)=$ $0, Y_{1}=0, \theta_{2}=0$, then

$$
\psi(t)=\frac{1}{s+1} \zeta(t)=J_{1} \frac{s}{s+1} y_{1}(t)-J_{2} \frac{1}{s+1} y_{2}(t)
$$

is a variable that we can calculate, and

$$
\begin{aligned}
\psi(t)= & \zeta(t)-\frac{1}{s+1} \dot{\zeta}(t)=\zeta(t)-\frac{1}{s+1}\left[Y_{2} \dot{y}_{1}(t)\right. \\
& \left.+Y_{3} y_{2}(t)+Y_{4} u(t)+Y_{5} \phi_{2}\left(y_{2}(t)\right)\right] .
\end{aligned}
$$

Hence, for a nonsingular $J_{1}$ we obtain $x_{2}(t)=J_{1}^{-1} \tilde{\psi}(t)$, where

$$
\begin{aligned}
\tilde{\psi}(t) & =\psi(t)+Y_{2} \frac{s}{s+1} y_{1}(t)+\frac{1}{s+1}\left[Y_{3} y_{2}(t)\right. \\
& \left.+Y_{4} u(t)+Y_{5} \phi_{2}\left(y_{2}(t)\right)\right]+J_{2} y_{2}(t)
\end{aligned}
$$

can be calculated using filters. If $d_{1}(t) \neq 0$ and $d_{2}(t) \neq 0$, then these approaches lead to a reconstruction of unknown parameters and variables corrupted by noises, and robust estimation tools should be applied. Next, a control design has to be performed. A drawback of such solutions is also their computational complexity comparing to (3), (4), (5), where just additional adaptation algorithms (observers or filters) are introduced to calculate $\hat{\theta}_{1}(t)$ and $\hat{\theta}_{2}(t)$ in order to compensate the influence of $\theta_{1}$ and $\theta_{2}$.

It is worth noting that a complexity of (3), (4), (5) comes from another side. Initially the system (2) is delay-free (the state $x(t) \in \mathbb{R}^{2 n+p}$ ), then (3), (4), (5) introduces the delay $h$ and transforms the system into the retarded type time-delay dynamics [6] (the state function is from $C_{[-h, 0]}^{2 n+p}$ ), while for the stability analysis we will perform below an additional transformation of the closed-loop system to the neutral type (with the state from $\mathbb{W}_{[-h, 0]}^{1, \infty}$ ). Therefore, (3), (4), (5) needs a rather sophisticated analysis, but it allows a simple realization.

The restrictions on selection of the control and adaptation gains, and the conditions to check, are given in the following theorem.

Theorem 8. Let Assumption 1 be satisfied. If for given $K_{i}, F_{i}$ with $i=\overline{1,4}$ and $S_{i}$ with $i=1,5$ the system of linear matrix inequalities

$$
\begin{gathered}
Q \leq 0, P=P^{\top}>0, \alpha>0, \beta>0, \delta>0, \eta>0, \\
\mathcal{M}^{\top} \mathcal{M} \leq \rho I_{s_{1}+s_{2}}, 4 \frac{e^{-\varpi h}}{h^{2}} I_{n} \geq \alpha \mathcal{B}^{\top} \mathcal{B} \\
\mathcal{S}=\mathcal{G}^{\top} P+q h^{2} \mathcal{G}^{\top} \Gamma^{\top} \Gamma \mathcal{A}, \\
S_{2}=-q h^{2} \mathcal{G}^{\top} \Gamma^{\top} \Gamma \mathcal{B}, \mathcal{S}_{4}=q h^{2} \mathcal{G}^{\top} \Gamma^{\top} \Gamma \mathcal{M} \\
Q=\left[\begin{array}{ccc}
Q_{11} & N & N \\
N^{\top} & M-\alpha q I_{3 n+p} & M \\
N^{\top} & M & M-\beta I_{3 n+p} \\
0 & 0 & 0 \\
N^{\top} & M & M \\
0 & 0 & 0 \\
0 & N & 0 \\
0 & M & 0 \\
0 & M & -S_{5} \\
Q_{44} & M-\left(\eta-q h^{2}\right) I_{3 n+p} & 0 \\
0 & 0 & -\delta I_{r}
\end{array}\right], \\
-S_{5}^{\top} \\
Q_{11}=P \mathcal{A}+\mathcal{A}^{\top} P+q h^{2} \mathcal{A}^{\top} \Gamma^{\top} \Gamma \mathcal{A}+\rho \beta C^{\top} \Upsilon C+\varpi P, \\
Q_{44}=-2 S_{5}+\left(q h^{2}\left|B_{1}\right|^{2} \bar{\Omega}^{2}+\varpi\right) I_{r}, \\
N=P+q h^{2} \mathcal{A}^{\top} \Gamma^{\top} \Gamma, M=q h^{2} \Gamma^{\top} \Gamma,
\end{gathered}
$$

is feasible with respect to $P, \alpha, \beta, \delta$ and $\eta$ for some $q>0$, $\varpi>0$ and $\rho>0$, where

$$
\begin{aligned}
& \mathcal{A}=\left[\begin{array}{cccc}
0 & I_{n} & 0 & 0 \\
A_{21}-B_{1} K_{1} & A_{22}-h B_{1} K_{2} & A_{23}-B_{1} K_{3} & B_{1} K_{1} \\
A_{31}-B_{2} K_{1} & A_{32}-h B_{2} K_{2} & A_{33}-B_{2} K_{3} & B_{2} K_{1} \\
F_{1} & h F_{2} & F_{3} & -F_{1}
\end{array}\right] \\
& \mathcal{B}=\left[\begin{array}{c}
0 \\
B_{1} K_{2} \\
B_{2} K_{2} \\
-F_{2}
\end{array}\right], \mathcal{G}=\left[\begin{array}{c}
0 \\
B_{1} \\
B_{2} \\
0
\end{array}\right], \mathcal{D}=\left[\begin{array}{c}
0 \\
I_{n} \\
I_{p} \\
0
\end{array}\right] \\
& \mathcal{M}=\left[\begin{array}{cc}
0 & 0 \\
L_{11} & L_{12}-B_{1} K_{4} \\
L_{21} & L_{22}-B_{2} K_{4} \\
0 & F_{4}
\end{array}\right], C=\left[\begin{array}{cccc}
I_{n} & 0 & 0 & 0 \\
0 & I_{n} & 0 & 0 \\
0 & 0 & I_{p} & 0
\end{array}\right] \text {, } \\
& \mathcal{S}=\left[\begin{array}{llll}
S_{1} & h S_{2} & S_{3} & -S_{1}
\end{array}\right], \mathcal{S}_{4}=\left[\begin{array}{ll}
0 & S_{4}
\end{array}\right] \text {, } \\
& \Gamma=\left[\begin{array}{llll}
0 & I_{n} & 0 & 0
\end{array}\right] .
\end{aligned}
$$

Then the system (2) with the control (3) and adaptive laws (4), (5) is practically ISS.

Proof: Following the ideas of [8], [9] note that $y_{1}(t-h)=$ $y_{1}(t)-h \dot{y}_{1}(t)+R(t)$ for $R(t)=\int_{t-h}^{t}(s-t+h) \ddot{y}_{1}(s) d s$, which can be easily checked by applying integration by parts, then the expressions for (3), (4), (5) can be represented as follows:

$$
\begin{aligned}
& u(t)=-K_{1} y_{1}(t)-h K_{2} \dot{y}_{1}(t)+K_{2} R(t) \\
&-K_{3} y_{2}(t)-K_{4} \phi_{2}\left(y_{2}(t)\right) \\
&+K_{1} \hat{\theta}_{1}(t)-\Omega(t) \hat{\theta}_{2}(t) \\
&=-K_{1} x_{1}(t)-h K_{2} x_{2}(t)+K_{2} R(t) \\
&-K_{3} x_{3}(t)-K_{4} \phi_{2}\left(x_{3}(t)\right) \\
&+K_{1} \tilde{\theta}_{1}(t)-\Omega(t) \hat{\theta}_{2}(t), \\
& \dot{\hat{\theta}}_{1}(t)= F_{1} y_{1}(t)+h F_{2} \dot{y}_{1}(t)-F_{2} R(t) \\
&+F_{3} y_{2}(t)+F_{4} \phi_{2}\left(y_{2}(t)\right)-F_{1} \hat{\theta}_{1}(t) \\
&= F_{1} x_{1}(t)+h F_{2} x_{2}(t)-F_{2} R(t) \\
&+F_{3} x_{3}(t)+F_{4} \phi_{2}\left(x_{3}(t)\right)-F_{1} \tilde{\theta}_{1}(t), \\
& \Omega^{\top}(t)\left[S_{1} y_{1}(t)+h S_{2} \dot{y}_{1}(t)-S_{2} R(t)\right.\left.+S_{3} y_{2}(t)+S_{4} \phi_{2}\left(y_{2}(t)\right)-S_{1} \hat{\theta}_{1}(t)\right] \\
&-S_{5} \hat{\theta}_{2}(t) \\
& \dot{\hat{\theta}}_{2}(t)=\Omega^{\top}(t)\left[S_{1} x_{1}(t)+h S_{2} x_{2}(t)-S_{2} R(t)\right. \\
&\left.+S_{3} x_{3}(t)+S_{4} \phi_{2}\left(x_{3}(t)\right)-S_{1} \tilde{\theta}_{1}(t)\right] \\
&-S_{5} \hat{\theta}_{2}(t),
\end{aligned}
$$

where we substituted the expressions of the outputs $y_{1}(t)$ and $y_{2}(t)$ (note that $\dot{y}_{1}(t)=x_{2}(t)$ since $\theta_{1}$ is constant), and $\tilde{\theta}_{1}(t)=\hat{\theta}_{1}(t)-\theta_{1}$ is the adaptation error for $\hat{\theta}_{1}(t)$. Similarly we define $\tilde{\theta}_{2}(t)=\hat{\theta}_{2}(t)-\theta_{2}$ as the adaptation error for $\hat{\theta}_{2}(t)$, and for $z(t)=\left[x^{\top}(t) \tilde{\theta}_{1}^{\top}(t)\right]^{\top}$ the closed loop dynamics take the form:

$$
\begin{aligned}
\dot{z}(t)= & \mathcal{A} z(t)+\mathcal{B} R(t)-\mathcal{G} \Omega(t) \tilde{\theta}_{2}(t) \\
& +\mathcal{M} \phi(C z(t))+\mathcal{D} d(t), \\
\dot{\tilde{\theta}}_{2}(t)= & \Omega^{\top}(t)\left[\mathcal{S} z(t)-S_{2} R(t)\right. \\
& \left.+\mathcal{S}_{4} \phi(C z(t))\right]-S_{5} \tilde{\theta}_{2}(t)-S_{5} \theta_{2},
\end{aligned}
$$

where all matrices are defined in the formulation of the theorem. Obviously, due to presence of $R(t)$, which contains the state derivative $\Gamma \dot{z}(s)$ with $s \in[t-h, t]$, the system (7) is of neutral type, then $z_{t} \in \mathbb{W}_{[-h, 0]}^{1, \infty}$ is the state together with $\tilde{\theta}_{2}(t) \in \mathbb{R}^{r}$. Then, in order to analyze ISS property of (7) with 
respect to the inputs $d(t)$ and $\theta_{2}$, let us consider a LyapunovKrasovskii functional candidate:

$$
\begin{gathered}
V\left(z_{t}, \dot{z}_{t}, \tilde{\theta}_{2}(t)\right)=z^{\top}(t) P z(t)+q W\left(z_{t}, \dot{z}_{t}\right)+\tilde{\theta}_{2}^{\top}(t) \tilde{\theta}_{2}(t), \\
W\left(z_{t}, \dot{z}_{t}\right)=\int_{t-h}^{t} e^{\varpi(s-t)}(s-t+h)^{2} \dot{z}^{\top}(s) \Gamma^{\top} \Gamma \dot{z}(s) d s,
\end{gathered}
$$

where $P=P^{\top}>0$ and $q>0$ are solutions of (6) and $\varpi>0$ (if we would substitute the expression of $\Gamma \dot{z}(s)$ from (7), then $V$ will be a function of the disturbance $d$, hence, the neutral interpretation of (2), (3), (4), (5) is obligatory). For $W(t)=W\left(z_{t}, \dot{z}_{t}\right)$ a direct computation gives:

$$
\begin{aligned}
\dot{W}(t)= & -2 \int_{t-h}^{t} e^{\varpi(s-t)}(s-t+h) \dot{z}^{\top}(s) \Gamma^{\top} \Gamma \dot{z}(s) d s \\
& +h^{2} \dot{z}^{\top}(t) \Gamma^{\top} \Gamma \dot{z}(t)-\varpi W(t),
\end{aligned}
$$

and applying Jensen's inequality [6] we obtain:

$\int_{t-h}^{t} e^{\varpi(s-t)}(s-t+h) \dot{z}^{\top}(s) \Gamma^{\top} \Gamma \dot{z}(s) d s \geq 2 \frac{e^{-\varpi h}}{h^{2}} R^{\top}(t) R(t)$,

then

$$
\dot{W}(t) \leq h^{2} \dot{z}^{\top}(t) \Gamma^{\top} \Gamma \dot{z}(t)-4 \frac{e^{-\varpi h}}{h^{2}} R^{\top}(t) R(t)-\varpi W(t) .
$$

The full derivative of $V(t)=V\left(z_{t}, \dot{z}_{t}, \tilde{\theta}_{2}(t)\right)$ for (7) can now be estimated as follows:

$$
\begin{gathered}
\dot{V}(t) \leq z^{\top}(t) P \dot{z}(t)+\dot{z}^{\top}(t) P z(t)+q h^{2} \dot{z}^{\top}(t) \Gamma^{\top} \Gamma \dot{z}(t) \\
-4 q \frac{e^{-\varpi h}}{h^{2}} R^{\top}(t) R(t)-q \varpi W(t)+2 \tilde{\theta}_{2}^{\top}(t) \dot{\tilde{\theta}}_{2}(t) \\
\leq \xi^{\top}(t) \mathcal{Q}(t) \xi(t)-\varpi V(t)-q R^{\top}(t)\left(4 \frac{e^{-\varpi h}}{h^{2}}-\alpha \mathcal{B}^{\top} \mathcal{B}\right) R(t) \\
+\beta\left[\phi^{\top}(C z(t)) \mathcal{M}^{\top} \mathcal{M} \phi(C z(t))-\rho z^{\top}(t) C^{\top} \Upsilon C z(t)\right] \\
+\eta d^{\top}(t) d(t)+\delta \theta_{2}^{\top} \theta_{2} \\
+2 \tilde{\theta}_{2}^{\top}(t) \Omega^{\top}(t)\left[\left(\mathcal{S}-\mathcal{G}^{\top} P-q h^{2} \mathcal{G}^{\top} \Gamma^{\top} \Gamma \mathcal{A}\right) z(t)\right. \\
-\left(S_{2}+q h^{2} \mathcal{G}^{\top} \Gamma \Gamma^{\top} \Gamma \mathcal{B}\right) R(t) \\
\left.+\left(\mathcal{S}_{4}-q h^{2} \mathcal{G}^{\top} \Gamma^{\top} \Gamma \mathcal{M}\right) \phi(C z(t))\right],
\end{gathered}
$$

where

$$
\begin{array}{r}
\xi(t)=\left[z^{\top}(t) R^{\top}(t) \mathcal{B}^{\top} \phi^{\top}(C z(t)) \mathcal{M}^{\top} \tilde{\theta}_{2}^{\top}(t) d^{\top}(t) \mathcal{D}^{\top} \theta_{2}^{\top}\right]^{\top}, \\
\mathcal{Q}(t)=\left[\begin{array}{ccc}
Q_{11} & N & N \\
N^{\top} & M-\alpha q I_{3 n+p} & M \\
N^{\top} & M & M-\beta I_{3 n+p} \\
0 & 0 & 0 \\
N^{\top} & M & M \\
0 & 0 & 0 \\
0 & N & 0 \\
0 & M & 0 \\
0 & M & -S_{5} \\
\mathcal{E}(t) & 0 & 0 \\
0 & M-\left(\eta-q h^{2}\right) I_{3 n+p} & -\delta I_{r}
\end{array}\right], \\
-S_{5}^{\top} \\
\mathcal{E}(t)=-2 S_{5}+q h^{2} H^{\top}(t) H(t)+\varpi I_{r}, H(t)=\Gamma \mathcal{G} \Omega(t),
\end{array}
$$

where the matrices $M$ and $N$ are introduced in the formulation of the theorem, the parameters $\alpha, \beta, \delta$ and $\eta$ come from (6), and the only inequality used on the last step is

$$
\begin{gathered}
\tilde{\theta}_{2}^{\top}(t) H^{\top}(t) \Gamma \mathcal{D} d(t) \leq 0.5 \tilde{\theta}_{2}^{\top}(t) H^{\top}(t) \Gamma \Gamma^{\top} H(t) \tilde{\theta}_{2}(t) \\
\quad+0.5 d^{\top}(t) \mathcal{D}^{\top} \mathcal{D} d(t) \\
=0.5 \tilde{\theta}_{2}^{\top}(t) H^{\top}(t) H(t) \tilde{\theta}_{2}(t)+0.5 d^{\top}(t) \mathcal{D}^{\top} \mathcal{D} d(t) .
\end{gathered}
$$

Due to the equalities imposed in (6), the expression in square brackets, which is multiplied from the left by $\tilde{\theta}_{2}^{\top}(t) \Omega^{\top}(t)$, equals zero, and the term $R^{\top}(t)\left(\alpha \mathcal{B}^{\top} \mathcal{B}-4 \frac{e^{-\varpi h}}{h^{2}} I_{n}\right) R(t)$ is non-positive. Note that according to Assumption 1 . $\phi^{\top}(C z(t)) \phi(C z(t)) \leq z^{\top}(t) C^{\top} \Upsilon C z(t)$, and from $\mathcal{M}^{\top} \mathcal{M} \leq$ $\rho I_{s_{1}+s_{2}}$ we get that

$$
\phi^{\top}(C z(t)) \mathcal{M}^{\top} \mathcal{M} \phi(C z(t)) \leq \rho z^{\top}(t) C^{\top} \Upsilon C z(t) .
$$

Therefore, the upper estimate on the derivative of the Lyapunov-Krasovskii functional can be rewritten as follows:

$$
\dot{V}(t) \leq \xi^{\top}(t) Q \xi(t)-\varpi V(t)+\eta d^{\top}(t) d(t)+\delta \theta_{2}^{\top} \theta_{2},
$$

where we used the fact that $|H(t)| \leq\left|B_{1}\right| \bar{\Omega}$ from Assumption 11. then

$$
\mathcal{E}(t) \leq-2 S_{5}+\left(q h^{2}\left|B_{1}\right|^{2} \bar{\Omega}^{2}+\varpi\right) I_{r}
$$

Finally, since by the conditions of the theorem $Q \leq 0$, we obtain

$$
\dot{V}(t) \leq-\varpi V(t)+\eta d^{\top}(t) d(t)+\delta \theta_{2}^{\top} \theta_{2},
$$

which implies that $V$ is an ISS Lyapunov-Krasovskii functional for (7) and the system possesses ISS property with respect to the inputs $d(t)$ and $\theta_{2}$ (practical ISS with respect to the input $d(t))$ due to Theorem 4 provided that there exist $\alpha_{1}, \alpha_{2}, \alpha_{1}^{\prime}, \alpha_{2}^{\prime} \in \mathcal{K}_{\infty}$ such that

$$
\begin{gathered}
\alpha_{1}(|z(t)|)+\alpha_{1}^{\prime}\left(\left|\tilde{\theta}_{2}(t)\right|\right) \leq V\left(z_{t}, \dot{z}_{t}, \tilde{\theta}_{2}(t)\right) \\
\leq \alpha_{2}\left(\left\|z_{t}\right\| \mathbb{W}\right)+\alpha_{2}^{\prime}\left(\left|\tilde{\theta}_{2}(t)\right|\right)
\end{gathered}
$$

for all $z_{t} \in \mathbb{W}_{[-h, 0]}^{1, \infty}$ and $\tilde{\theta}_{2}(t) \in \mathbb{R}^{r}$. Obviously, $\alpha_{1}(s)=$ $\lambda_{\min }(P) s^{2}, \alpha_{1}^{\prime}(s)=\alpha_{2}^{\prime}(s)=s^{2}$, and in order to evaluate $\alpha_{2}$ let us consider

$$
\begin{gathered}
W\left(z_{t}, \dot{z}_{t}\right) \leq \int_{t-h}^{t}(s-t+h)^{2} \dot{z}^{\top}(s) \Gamma^{\top} \Gamma \dot{z}(s) d s \\
\leq h^{2} \int_{t-h}^{t} \dot{z}^{\top}(s) \Gamma^{\top} \Gamma \dot{z}(s) d s \\
\leq h^{3} \sup _{s \in[t-h, t]} \dot{z}^{\top}(s) \Gamma^{\top} \Gamma \dot{z}(s) \leq h^{3}\left\|\dot{z}_{t}\right\|^{2} \leq h^{3}\left\|z_{t}\right\|_{\mathbb{W}}^{2},
\end{gathered}
$$

then $\alpha_{2}(s)=\lambda_{\max }(P) s^{2}+q h^{3} s^{2}$.

The conditions of the theorem connect the control parameters to be tuned (the gains $K_{i}, F_{i}, S_{i}$ and the admissible delay $h)$, the auxiliary constants $(q>0, \varpi>0, \rho>0)$ and the variables of linear matrix inequalities $(Q \leq 0, P>0$, $\alpha>0, \beta>0, \delta>0$ and $\eta>0$ ), which are obtained applying numerical solvers to [6]. By arguments of [8], [9] it can be shown that the inequalities in (6) are always feasible for a sufficiently small value of $h$.

Remark 9. As it follows from the proof of Theorem 8 and the estimate (8) calculated for the derivative of the LyapunovKrasovskii functional $V$, if there is no disturbance and the parametric uncertainty is presented by the measurement bias only (i.e., $d=0$ and $\theta_{2}=0$ ), then the studied closedloop system is globally asymptotically stable. Therefore, the value of the bias $\theta_{1}$ can be exactly identified by the adjusted parameter $\hat{\theta}_{1}(t)$, and such an achievement is possible without imposing any excitation requirement since the bias value is contained in the output $y_{1}$ directly. 
Remark 10. The presentation above is given by factorizing the LMIs in the briefest way, which, however, may be more conservative. For example, taking

$$
\xi(t)=\left[z^{\top}(t) R^{\top}(t) \phi^{\top}(C z(t)) \tilde{\theta}_{2}^{\top}(t) d^{\top}(t) \theta_{2}^{\top}\right]^{\top}
$$

we obtain that if for given $K_{i}, F_{i}$ with $i=\overline{1,4}$ and $S_{i}$ with $i=\overline{1,5}$ the system of linear matrix inequalities

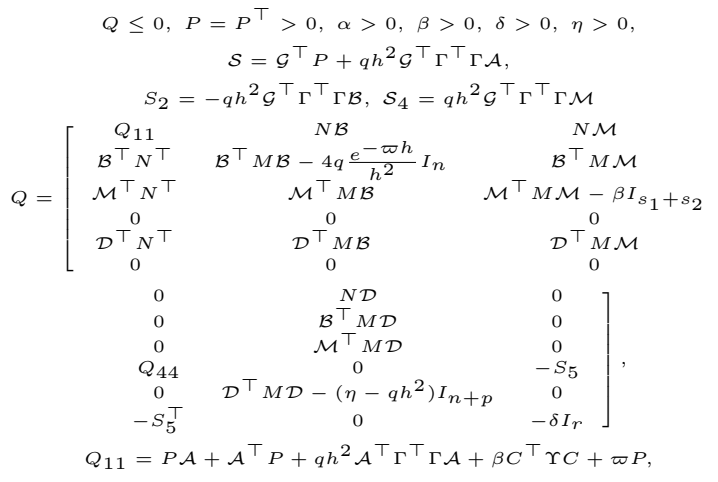

is feasible with respect to $P, \alpha, \beta, \delta$ and $\eta$ for some $q>0$ and $\varpi>0$ (where the meaning of other variables is the same as in the formulation of Theorem 8), then the system (2) with the control (3) and adaptive laws (4), (5) is also practically ISS. However, it is worth to stress a more nonlinear nature of (9) comparing with (6).

Remark 11. If appearance of an additional noise $v \in \mathcal{L}_{\infty}^{n+p}$ in the output measurements of (2) is assumed:

$$
y_{1}(t)=x_{1}(t)+\theta_{1}+v_{1}(t), \quad y_{2}(t)=x_{3}(t)+v_{2}(t)
$$

with $v(t)=\left[\begin{array}{ll}v_{1}^{\top}(t) & v_{2}^{\top}(t)\end{array}\right]^{\top} \in \mathbb{R}^{n+p}$, then it results in a linear perturbation of the control (3) (except for the nonlinearity $\phi_{2}\left(y_{2}(t)\right)$, but since it is supposed to be Lipschitz, the treatment of this term will not change the conclusion), whose influence can be masked by $d$. The adaptation algorithms (4) and (5) contain already the negative robustifying feedbacks proportional to $\hat{\theta}_{1}(t)$ and $\hat{\theta}_{2}(t)$, respectively (they are included to counteract the disturbance $d$ ). Hence, an additive measurement noise $v$ will not impact stability, and the ISS property can be proven for the closed-loop system with respect to both, the state perturbation $d$ and the measurement noise $v$, by applying the same arguments.

In Theorem 8 it is assumed that the control and adaptation gains $K_{i}, F_{i}, S_{i}$ are already given. A way to obtain them consists in solution of the stabilization problem under assumption that the state is measured, and next to look for the delay $h$ that verifies the above restrictions. However, by introducing additional mild restrictions we can reformulate the conditions of Theorem 8 by considering the control and adaptation gains $K_{i}, F_{i}, S_{i}$ as solutions of LMIs:

Corollary 12. Let Assumption 1 be satisfied for $\Upsilon>0$, if the system of linear matrix inequalities

$$
\begin{aligned}
& \tilde{Q} \leq 0, P^{-1}=P^{-\top} \geq 0, \alpha>0, \beta>0, \delta>0, \eta>0, \\
& {\left[\begin{array}{cc}
\rho I_{s_{1}+s_{2}} & \mathcal{M}^{\top} \\
\mathcal{M} & I_{3 n+p}
\end{array}\right] \geq 0,\left[\begin{array}{cc}
I_{3 n+p} & P^{-1} C^{\top} \\
C P^{-1} & \frac{1}{\rho} \Upsilon^{-1}
\end{array}\right] \geq 0,} \\
& {\left[\begin{array}{cc}
2 P^{-1}-\frac{\alpha}{4} e^{\varpi h} \Delta^{-1} & \mathcal{U}^{\top} \mathcal{G}^{\top}-\mathcal{W}^{\top} \mathcal{I}^{\top} \\
\mathcal{G} \mathcal{U}-\mathcal{I} \mathcal{W} & I_{3 n+p}
\end{array}\right] \geq 0} \\
& \Delta=c \operatorname{diag}\left[I_{n}, c^{-1} I_{n}, I_{p}, I_{n}\right], \\
& \Sigma=\mathcal{G}^{\top}+q h^{2} B_{1}^{\top}\left(\Gamma \mathcal{A}_{0} P^{-1}-B_{1} \mathcal{U}\right), \\
& \Sigma_{2}=-q h^{2} B_{1}^{\top} B_{1} \mathcal{U}_{2},\left[\begin{array}{ll}
0 & S_{4}
\end{array}\right]=q h^{2} B_{1}^{\top}\left[\begin{array}{ll}
L_{11} & L_{12}-B_{1} K_{4}
\end{array}\right] \\
& \tilde{Q}=\left[\begin{array}{cccc}
\tilde{Q}_{11} & I_{3 n+p} & I_{3 n+p} & 0 \\
I_{3 n+p} & -\alpha q I_{3 n+p} & 0 & 0 \\
I_{3 n+p} & 0 & -\beta I_{3 n+p} & 0 \\
0 & 0 & & 0
\end{array}\right. \\
& \tilde{Q}=\mid \begin{array}{cccc}
I_{3 n+p} & 0 & -\beta I_{3 n+p} & 0 \\
0 & 0 & 0 & Q_{44} \\
I_{3 n+p} & 0 & 0 & 0 \\
0 & 0 & 0 & -S_{5}^{\top}
\end{array} \\
& {\left[\begin{array}{cccc}
0 & 0 & 0 & -S_{5}^{\top} \\
\tilde{Q}_{17}^{\top} & -\Gamma & -\Gamma & 0
\end{array}\right.} \\
& \left.\begin{array}{ccc}
I_{3 n+p} & 0 & \tilde{Q}_{17} \\
0 & 0 & -\Gamma^{\top} \\
0 & 0 & -\Gamma^{\top} \\
0 & -S_{5} & 0 \\
-\left(\eta-q h^{2}\right) I_{3 n+p} & 0 & -\Gamma^{\top} \\
0 & -\delta I_{r} & 0 \\
-\Gamma & 0 & -\frac{1}{q h^{2}} I_{n}
\end{array}\right], \\
& \tilde{Q}_{11}=\mathcal{A}_{0} P^{-1}+P^{-1} \mathcal{A}_{0}^{\top}-\mathcal{G} \mathcal{U}-\mathcal{U}^{\top} \mathcal{G}^{\top} \\
& +\mathcal{I} \mathcal{W}+\mathcal{W}^{\top} \mathcal{I}^{\top}+\beta I_{3 n+p}+\varpi P^{-1} \\
& \tilde{Q}_{17}=-P^{-1} \mathcal{A}_{0}^{\top} \Gamma^{\top}+\mathcal{U}^{\top} B_{1}^{\top}, Q_{44}=-2 S_{5}+\left(q h^{2}\left|B_{1}\right|^{2} \bar{\Omega}^{2}+\varpi\right) I_{r},
\end{aligned}
$$

is feasible with respect to $P^{-1}, \mathcal{U}, \mathcal{W}, \Sigma, K_{4}, F_{4}, S_{4}, S_{5}, \alpha$, $\beta, \eta$ and $\delta$ for some given $q>0, c>0, \varpi>0, \mu>0$ and $\rho>0$, where

$$
\mathcal{A}_{0}=\left[\begin{array}{cccc}
0 & I_{n} & 0 & 0 \\
A_{21} & A_{22} & A_{23} & 0 \\
A_{31} & A_{32} & A_{33} & 0 \\
0 & 0 & 0 & 0
\end{array}\right], \mathcal{I}=\left[\begin{array}{c}
0 \\
0 \\
0 \\
I_{n}
\end{array}\right],
$$

and the matrices $\mathcal{B}, \mathcal{G}, \mathcal{D}, \mathcal{M}, C$ and $\Gamma$ are defined in Theorem 8. Then for

$$
\begin{gathered}
{\left[\begin{array}{cccc}
K_{1} & h K_{2} & K_{3} & K_{5}
\end{array}\right]=\mathcal{U P},} \\
{\left[\begin{array}{cccc}
F_{1} & h F_{2} & F_{3} & F_{5}
\end{array}\right]=\mathcal{W} P,} \\
{\left[\begin{array}{llll}
S_{1} & h S_{2} & S_{3} & S_{6}
\end{array}\right]=\Sigma P,}
\end{gathered}
$$

the system (2) with the control and adaptive laws

$$
\begin{gathered}
u(t)=-\left(K_{1}+K_{2}\right) y_{1}(t)+K_{2} y_{1}(t-h) \\
-K_{3} y_{2}(t)-K_{4} \phi_{2}\left(y_{2}(t)\right)-K_{5} \hat{\theta}_{1}(t)-\Omega(t) \hat{\theta}_{2}(t), \\
\dot{\hat{\theta}}_{1}(t)=\quad\left(F_{1}+F_{2}\right) y_{1}(t)-F_{2} y_{1}(t-h) \\
\quad+F_{3} y_{2}(t)+F_{4} \phi_{2}\left(y_{2}(t)\right)+F_{5} \hat{\theta}_{1}(t), \\
\left.\dot{\hat{\theta}}_{2}(t)=\quad \begin{array}{l}
\Omega^{\top}(t)\left[\left(S_{1}+S_{2}\right) y_{1}(t)-S_{2} y_{1}(t-h)\right. \\
\\
\end{array} \quad S_{3} y_{2}(t)+S_{4} \phi_{2}\left(y_{2}(t)\right)+S_{6} \hat{\theta}_{1}(t)\right]-S_{5} \hat{\theta}_{2}(t),
\end{gathered}
$$

is practically ISS.

Proof: Note that

$$
\mathcal{A}=\mathcal{A}_{0}-\mathcal{G} \mathscr{K}+\mathcal{I} \mathcal{F}
$$

where $\mathscr{K}=\left[\begin{array}{llll}K_{1} & h K_{2} & K_{3} & K_{5}\end{array}\right] \quad$ and $\mathcal{F}=\left[\begin{array}{llll}F_{1} & h F_{2} & F_{3} & F_{5}\end{array}\right]$ contain the searched gains. Then with these designations, by applying Schur complement to the matrix $Q$, by multiplying it 
from the left and right by a positive definite matrix $\operatorname{diag}\left\{P^{-1}, I_{3 n+p}, I_{3 n+p}, I_{3 n+p}, I_{3 n+p}, I_{3 n+p}, I_{n}\right\}$, using the identity

$$
\mathcal{A} P^{-1}=\mathcal{A}_{0} P^{-1}-\mathcal{G U}+\mathcal{I} \mathcal{W},
$$

where $\mathcal{W}=\mathcal{F} P^{-1}$ and $\mathcal{U}=\mathscr{K} P^{-1}$ are decision variables, and introducing an auxiliary restriction

$$
\left[\begin{array}{cc}
I_{3 n+p} & P^{-1} C^{\top} \\
C P^{-1} & \frac{1}{\rho} \Upsilon^{-1}
\end{array}\right] \geq 0
$$

we obtain $\tilde{Q} \leq 0$, where we used the facts that $\Gamma \mathcal{I}=0$ and $\Gamma \mathcal{G}=B_{1}$. Two equalities given in (6) take the form

$$
\Sigma=\mathcal{G}^{\top}+q h^{2} B_{1}^{\top}\left(\Gamma \mathcal{A}_{0} P^{-1}-B_{1} \mathcal{U}\right)
$$

and $\Sigma_{2}=-q h^{2} B_{1}^{\top} B_{1} \mathcal{U}_{2}$ from $\left[\begin{array}{llll}0 & S_{2} & 0 & 0\end{array}\right] P^{-1}=$ $-q h^{2} B_{1}^{\top} B_{1}\left[\begin{array}{llll}0 & K_{2} & 0 & 0\end{array}\right] P^{-1}$, where $\Sigma=\mathcal{S} P^{-1}$ with $\mathcal{S}=\left[\begin{array}{llll}S_{1} & h S_{2} & S_{3} & S_{6}\end{array}\right]$ as before. Using Schur complement the inequality $\mathcal{M}^{\top} \mathcal{M} \leq \rho I_{s_{1}+s_{2}}$ can be represented as

$$
\left[\begin{array}{cc}
\rho I_{s_{1}+s_{2}} & \mathcal{M}^{\top} \\
\mathcal{M} & I_{3 n+p}
\end{array}\right] \geq 0
$$

Finally, for the inequality $4 \frac{e^{-\varpi h}}{h^{2}} I_{n} \geq \alpha \mathcal{B}^{\top} \mathcal{B}$, note that $\mathcal{B}=h^{-1}(\mathcal{G} \mathscr{K}-\mathcal{I F}) \Gamma^{\top}$, and this inequality takes the form $\frac{4}{\alpha} e^{-\varpi h} I_{n} \geq \Gamma(\mathcal{G} \mathscr{K}-\mathcal{I} \mathcal{F})^{\top}(\mathcal{G} \mathscr{K}-\mathcal{I} \mathcal{F}) \Gamma^{\top}$, which follows from

$$
\frac{4}{\alpha} e^{-\varpi h} \Delta \geq(\mathcal{G} \mathscr{K}-\mathcal{I F})^{\top}(\mathcal{G} \mathscr{K}-\mathcal{I F})
$$

Multiplying the latter by $P^{-1}$ from the left and the right, we get:

$$
\frac{4}{\alpha} e^{-\varpi h} P^{-1} \Delta P^{-1} \geq(\mathcal{G U}-\mathcal{I} \mathcal{W})^{\top}(\mathcal{G U}-\mathcal{I} \mathcal{W}),
$$

and using again Schur complement, it can be transformed to

$$
\left[\begin{array}{cc}
\frac{4}{\alpha} e^{-\varpi h} P^{-1} \Delta P^{-1} & \mathcal{U}^{\top} \mathcal{G}^{\top}-\mathcal{W}^{\top} \mathcal{I}^{\top} \\
\mathcal{G U}-\mathcal{I} \mathcal{W} & I_{3 n+p}
\end{array}\right] \geq 0
$$

As in [26], applying the inequality $P^{-1} \frac{4}{\alpha} e^{-\varpi h} \Delta P^{-1} \geq$ $2 P^{-1}-\frac{\alpha}{4} e^{\varpi h} \Delta^{-1}$ the latter inequality is satisfied provided that

$$
\left[\begin{array}{cc}
2 P^{-1}-\frac{\alpha}{4} e^{\varpi h} \Delta^{-1} & \mathcal{U}^{\top} \mathcal{G}^{\top}-\mathcal{W}^{\top} \mathcal{I}^{\top} \\
\mathcal{G U}-\mathcal{I} \mathcal{W} & I_{3 n+p}
\end{array}\right] \geq 0,
$$

which is necessary to prove.

The last important observation is that for the algorithms (3), (4), (5) the variables $\mathscr{K}, \mathcal{F}$ and $\mathcal{S}$ have an additional linear constraint (i.e., $K_{1}=-K_{5}, F_{1}=-F_{5}$ and $S_{1}=-S_{6}$ ), which is hard to formulate in terms of an LMI since $\mathscr{K}=\mathcal{U P}$, $\mathcal{W}=\mathcal{F} P$ and $\mathcal{S}=\Sigma P$, then a possible solution is to assume that the variable $\hat{\theta}_{1}(t)$ in the algorithms (11), (12), (13) enters with an independent gain.

Let us consider some results of application of the proposed robust adaptive output control.

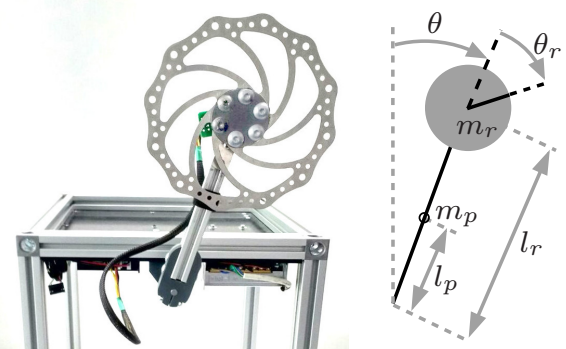

Figure 1: The inverted pendulum hardware and corresponding notations, see [19].

\section{Application to a nONLINEAR PENDUlum}

To illustrate the proposed approach, we consider an inverted pendulum stabilization problem, where the hardware setup used for experiments in this section has been previously reported in [19]. The equipment and corresponding notation are shown in Fig. 1

Define the state variable vector $x:=\left[\begin{array}{lll}\theta & \dot{\theta} & \dot{\theta}_{r}\end{array}\right]^{\top}$, where $\theta$ is the angle between the pendulum and the vertical, and $\theta_{r}$ is the angle of the reaction wheel with respect to the pendulum. Note that the reaction wheel velocity $\dot{\theta}_{r}$ is assumed to be available due to the equipment specifics. Derivations of the system dynamics and values of physical parameters can be found in [19] and are omitted here; the resulting model is given by

$$
\begin{aligned}
& \dot{x}_{1}=x_{2}, \\
& \dot{x}_{2}=a_{1} \sin \left(x_{1}\right)-b_{1} u, \\
& \dot{x}_{3}=-a_{1} \sin \left(x_{1}\right)+b_{2} u,
\end{aligned}
$$

where $a_{1}=49.9, b_{1}=1.4, b_{2}=30.9$, and $u$ is the motor current considered as the control input. Due to physical reasons, the angle $\theta$ (the state $x_{1}$ ) is bounded as $\left|x_{1}\right| \leq \frac{\pi}{2}$. The measurements are given by $y_{1}=x_{1}+\theta_{1}$ and $y_{2}=x_{3}$, where $\theta_{1}$ is the unknown constant bias of the pendulum position sensor. It is straightforward to verify that the model (14) can be written in the form (2) with $\Omega(t) \equiv 0$. The control goal is to drive the system to the origin and to estimate the bias $\theta_{1}$. In [19] a nonlinear velocity observer for the state $x_{2}$ has been proposed and combined with the state-feedback control law; however, only local convergence has been shown. In this section, we apply the control law (3), (4). Assumption 1 is satisfied with $\Upsilon=\operatorname{diag}[0.25,0,0]$. The LMI (9) is feasible with $h=0.01$ and $K_{1}=-102, K_{2}=-1698, K_{3}=-0.25$, $F_{1}=1, F_{3}=1.5 \cdot 10^{-4}$, and $K_{4}=F_{2}=F_{4}=0$, thus the conditions of Remark 10 are verified.

Some simulation results for this setup were presented in the preliminary work [12]. In the experiment, the bias is $\theta_{1}=0.125 \approx 7^{\circ}$. The results of the experimental pendulum stabilization and $\theta_{1}$ estimation with the control law (3), (4) are depicted in Fig. 2 for the estimated pendulum position $y_{1}-\hat{\theta}_{1}$ and the bias estimation $\hat{\theta}_{1}$. The experimental results for the reaction wheel velocity $y_{2}$ are depicted in Fig. 3, where the steady-state low-magnitude oscillations are attributed to the friction in the reaction wheel drive and compensation of external disturbances. The experimental results illustrate that the proposed delay-based control law stabilizes the system and 


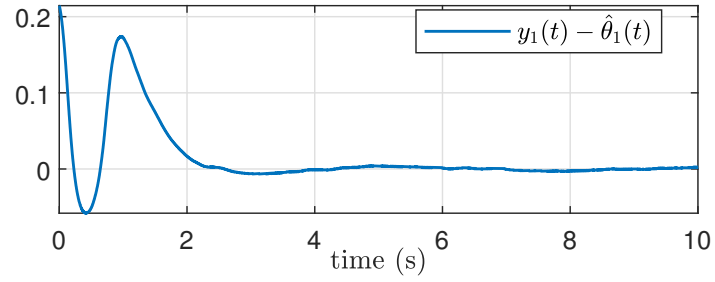

(a) The corrected pendulum position $y_{1}-\hat{\theta}_{1}$.

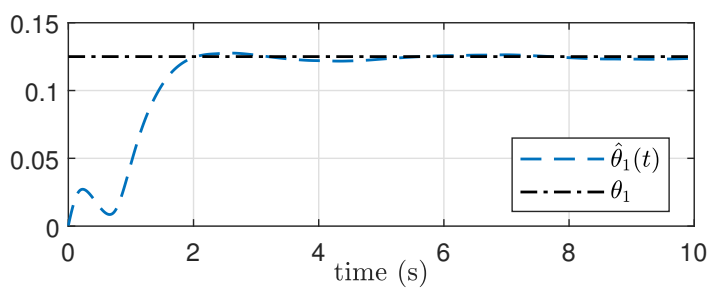

(b) The bias estimate $\hat{\theta}_{1}$ and the true bias value $\theta_{1}$.

Figure 2: Experimental stabilization of the inverted pendulum.

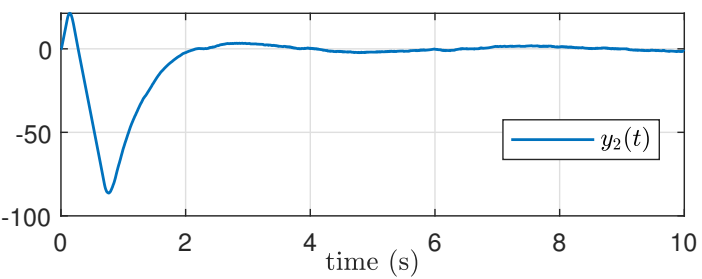

Figure 3: Experimental stabilization of the inverted pendulum: the reaction wheel velocity $y_{2}$.

allows for the bias estimation. The video of the experiment can be found here

\section{CONCLUSIONS}

Considering a Lipschitz nonlinear system, whose model contains external perturbation and uncertain parameters, while the measurements are available with a constant bias, the problem of robust output adaptive stabilization has been solved. Due to a severe uncertainty of the plant, the state reconstruction has been avoided by introducing artificial delays of the output in the feedback and adaptation algorithms. Applying the Lyapunov-Krasovskii approach, the conditions of practical ISS have been established, which are based on linear matrix inequalities. The efficacy of the proposed approach is demonstrated in experiments for an inertia wheel nonlinear pendulum. Extension of the proposed method to a more general class of systems is a direction of future research.

\section{REFERENCES}

[1] H. Khalil, Nonlinear Control. Upper Saddle River, New Jersey, 2015.

[2] S. Sastry and M. Bodson, Adaptive Control: Stability, Convergence and Robustness. London: Prentice-Hall, 1989.

[3] J.-P. Richard, "Time-delay systems: an overview of some recent advances and open problems," Automatica, vol. 39, pp. 1667-1694, 2003.

[4] X. Yang, X. Li, Q. Xi, and P. Duan, "Review of stability and stabilization for impulsive delayed systems," Mathematical Biosciences \& Engineering, vol. 15, no. 6, pp. 1495-1515, 2018.

[5] K. Gu, K. Kharitonov, and J. Chen, Stability of Time-Delay Systems. Boston: Birkhäuser, 2003.
[6] E. Fridman, Introduction to Time-Delay Systems: Analysis and Control. Basel: Birkhäuser, 2014.

[7] V. Kharitonov, S.-I. Niculescu, J. Moreno, and W. Michiels, "Static output feedback stabilization: necessary conditions for multiple delay controllers," IEEE Transactions on Automatic Control, vol. 50, no. 1 , pp. 82-86, 2005.

[8] E. Fridman and L. Shaikhet, "Delay-induced stability of vector secondorder systems via simple Lyapunov functionals," Automatica, vol. 74, pp. 288-296, 2016.

[9] E. Fridman and L. Shaikhet, "Stabilization by using artificial delays: An LMI approach," Automatica, vol. 81, pp. 429-437, 2017.

[10] D. Efimov, E. Fridman, W. Perruquetti, and J.-P. Richard, "Homogeneity of neutral systems and accelerated stabilization of a double integrator by measurement of its position," Automatica, vol. 118, no. 8, p. 109023 , 2020.

[11] A. Nekhoroshikh, D. Efimov, A. Polyakov, W. Perruquetti, I. Furtat, and E. Fridman, "On output-based accelerated stabilization of a chain of integrators: Implicit Lyapunov-Krasovskii functional approach," in Proc. 21rst IFAC World Congress, (Berlin), 2020.

[12] D. Efimov, S. Aranovskiy, E. Fridman, D. Sokolov, J. Wang, and A. Bobtsov, "Adaptive stabilization by delay with biased measurements," in Proc. 21rst IFAC World Congress, (Berlin), 2020.

[13] P. Pepe, "Adaptive output tracking for a class ofnonlinear time delay systems," International Journal of Adaptive Control and Signal Processing, vol. 18 , no. 6, pp. 489-503, 2004

[14] B. Mirkin and P.-O. Gutman, "Robust adaptive output-feedback tracking for a class of nonlinear time-delayed plants," IEEE Transactions on Automatic Control, vol. 55, no. 10, pp. 2418-2424, 2010.

[15] A. Selivanov, E. Fridman, and A. Fradkov, "Passification-based adaptive control: Uncertain input and output delays," Automatica, vol. 54, pp. 107-113, 2015.

[16] X. Zhang and W. Lin, "A K-filter-based adaptive control for nonlinear systems with unknown parameters in state and output equations," Automatica, vol. 105, pp. 186-197, 2019.

[17] X. Zhang and W. Lin, "Robust output feedback control of polynomial growth nonlinear systems with measurement uncertainty," Int. J. of Robust and Nonlinear Control, vol. 29, pp. 4562-4576, 2019.

[18] F. Mazenc, L. Burlion, and M. Malisoff, "Stabilization and robustness analysis for a chain of saturating integrators with imprecise measurements," IEEE Control Systems Letters, vol. 3, no. 2, pp. 428-433, 2019.

[19] S. Aranovskiy, I. Ryadchikov, R. Nikulchev, and D. Sokolov, "Differentiator-based velocity observer with sensor bias estimation: an inverted pendulum case study," in IFAC Symposium on Nonlinear Control Systems, September 2019.

[20] V. Kolmanovsky and V. Nosov, Stability of functional differential equations. San Diego: Academic, 1986.

[21] A. R. Teel, "Connections between Razumikhin-type theorems and the ISS nonlinear small gain theorem," IEEE Trans. Automat. Control, vol. 43, no. 7, pp. 960-964, 1998.

[22] P. Pepe and Z.-P. Jiang, "A Lyapunov-Krasovskii methodology for ISS and iISS of time-delay systems," Systems \& Control Letters, vol. 55, no. 12 , pp. 1006-1014, 2006.

[23] E. Fridman, M. Dambrine, and N. Yeganefar, "On input-to-state stability of systems with time-delay: A matrix inequalities approach," Automatica, vol. 44, no. 9, pp. 2364-2369, 2008.

[24] P. Pepe, I. Karafyllis, and Z.-P. Jiang, "Lyapunov-Krasovskii characterization of the input-to-state stability for neutral systems in Hale's form," Systems \& Control Letters, vol. 102, pp. 48-56, 2017.

[25] D. Efimov and E. Fridman, "Converse Lyapunov-Krasovskii theorem for ISS of neutralsystems in Sobolev spaces," Automatica, vol. 118, no. 8, p. $109042,2020$.

[26] J. Xu, Y. Niu, E. Fridman, and L. Fridman, "Finite frequency $h_{\infty}$ control of singularly perturbed Euler-Lagrange systems: An artificial delay approach," International Journal of Robust and Nonlinear Control, vol. 29, no. 2, pp. 353-374, 2019. 